In der folgenden Tafel finden sich die aus meinen Beobachtungen 'und aus den von Nijland mitgeteilten Phasen abgeleiteten Maxima, die eine unmittelbare Fortsetzung der A. N. 4506 (188.310) gegebenen Tafel bilden.

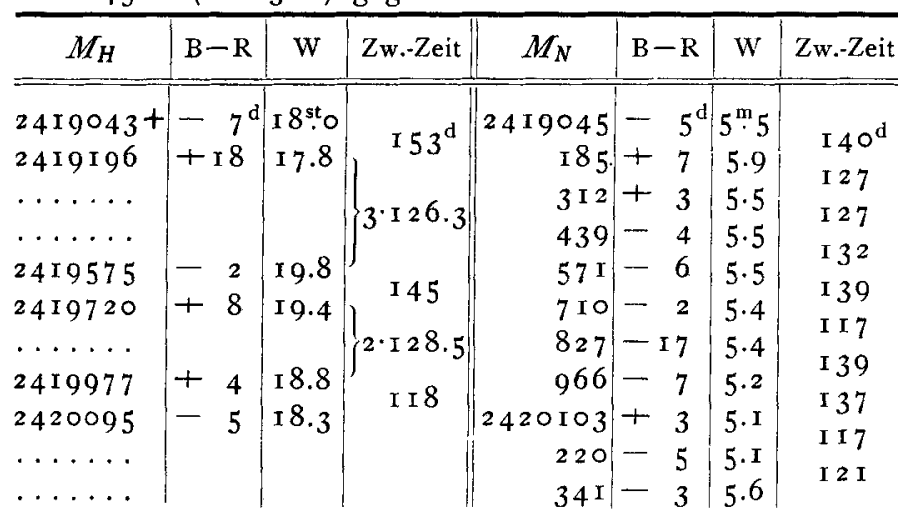

Die Unterschiede in den Epochen der gleichzeitig beobachteten Maxima überschreiten nicht die durch die geringe Dichte bedingte Unsicherheit. Die Maxima lassen sich ziemlich gut durch die folgende durch graphische Aus gleichung gefundene Formel darstellen:

$$
\begin{aligned}
& M=2419069.6+\mathrm{r} 26^{\mathrm{d}} \cdot 4 \cdot E+20^{\mathrm{d}} \sin \left(270^{\circ}+24^{\circ} E\right) \\
& m=M+69^{\mathrm{d}} 6 .
\end{aligned}
$$

Nach dieser Formel ergeben sich die folgenden in die Beobachtungszeit fallenden Maxima, deren Abweichung $(B-R)$ oben eingetragen ist.

\begin{tabular}{r|r|r}
\multicolumn{3}{|c}{ Berechnete Maxima: } \\
2419049.6 & 2419577.3 & 2420100.4 \\
177.7 & 711.6 & 225.4 \\
309.0 & 844.2 & 343.6 \\
442.6 & 973.0 &
\end{tabular}

Die Differenzen sind im allgemeinen klein und weisen keinen erkennbaren Gang auf.

Betrachten wir die Minima, so ergibt sich in ähnlicher

\begin{tabular}{|c|c|c|c|c|c|c|c|}
\hline$m_{H}$ & $B-R$ & w & Zw.-Zeit & $m_{N}$ & $B-R$ & W & $Z_{w}-Z_{\text {eit }}$ \\
\hline 2459128 & $+9^{d}$ & $I^{\text {st }} 4$ & & 2419122 & $+3^{\circ}$ & $6^{\mathrm{m}} \cdot 8$ & \\
\hline 2419246 & -1 & 3.2 & & 247 & & 6.5 & \\
\hline - & & & & 394 & +15 & 6.8 & 7 \\
\hline & & & $3^{-1} 34.7$ & 514 & +2 & 6.6 & $\begin{array}{l}0 \\
6\end{array}$ \\
\hline 2419650 & +3 & 2.4 & & 650 & +3 & 6.7 & \\
\hline & & & & 771 & -10 & 6.9 & 8 \\
\hline . & & & 30 & 909 & -5 & 6.7 & 10 \\
\hline 2420040 & -3 & $5 \cdot 3$ & $?$ & 2420032 & $-1 \mathrm{I}$ & 6.8 & 25 \\
\hline 2420164 & --6 & 3.4 & 124 & I 55 & $-\times 5$ & 6.9 & \\
\hline$\ldots \ldots$ & & & & 294 & $-\quad I$ & 6.7 & 39 \\
\hline
\end{tabular}
Weise folgende Tafel:
Wie die Rubrik $\mathrm{B}-\mathrm{R}$ lehrt, ist die Darstellung der Minima durch die gegebene Formel weniger gut als die der Maxima, doch immerhin noch befriedigend.

Die berechneten Minima sind folgende:

\begin{tabular}{r|r|r}
24 19119.2 & 2419646.9 & 2420042.6 \\
247.3 & 781.2 & I70.0 \\
378.6 & 913.8 & 295.0 \\
512.2 & &
\end{tabular}

Die Darstellung der Lichtkurve von W Cygni durch die mittlere Periode von $\mathrm{I}^{2} 6^{\mathrm{d}} \cdot 4$ mit einem sinus-Glied ist verhältnismäßig gut; sie gilt aber erst von dem Maximum 24 ×9049.6 an. Vorher, also vor der Epoche o meiner Skala, galt die von $N_{i j l a n d}$ in seiner früheren Veröffentlichung gegebene Formel:

$$
M=2418282+254^{\mathrm{d}} \cdot E .
$$

Da ich erst im Jahre r 9 Io mit der Beobachtung dieses Sternes begann, so war ich von vornherein auf die I 26 -tägige Periode gekommen, und ich konnte die Unstimmigkeit nicht erklären, daß die Beobachtungen des Jahres 19 I0, besonders im September, sich nicht durch sie darstellen ließen. Meine Beobachtungen vom April r 9 ro zeigen den Veränderlichen im Anstieg zum Maximum, das ja nach Nijland auch 2418795 eingetreten ist. Aber im August und September 1910 sah ich den Veränderlichen ziemlich schwach, und erst im Januar Ig I wieder im Maximum. Nach Nijland blieb der Stern zwischen den Maxima 2418795. und 2419045 von $24 \mathrm{I} 8800$ bis 980 nahezu konstant $\left(=6^{\mathrm{m}} \cdot 9\right)$. Diese lange Zeit unveränderter Helligkeit scheint also den Übergang von der einen Art des Lichtwechsels zur anderen zu bilden, nachdem der Stern in den Jahren 1907-08 eher einen unregelmäßigen Eindruck gemacht hatte.

Die jetzige Periode ist fast genau die Hälfte der unmittelbar vorhergehenden Periodenlänge; eine ähnliche Periodenlänge ( $130^{\mathrm{d}} 8$ ) ist bereits früher einmal beobachtet worden. ${ }^{1}$ ) Die wechselnden Periodenlängen scheinen nie lange anzuhalten, und es wäre ein interessantes Problem, der Frage nachzugehen, wie astrophysikalisch ein solcher Wechsel zu erklären wäre. Jedenfalls bedarf W Cygni weiter dringender Überwachung.

Im Maximum erreichte nach meinet Beobachtungen der Veränderliche durchschnittlich die Helligkeit $18^{\text {st }} 8$, während er im Minimum auf $3^{\text {sto }}$ hinabsank; die Schwankung seiner Helligkeit erreichte also mehr als I 5 Stufen meiner Skala und nach den Beobachtungen Nijlands 1.3.3 Größenklassen

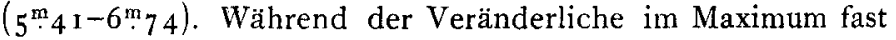
den Vergleichstern $a$ erreicht, sinkt er im Minimum bis auf die Helligkeit von $e$ herab.

Gnadenfrei, I9 I 4 Nov. r 9.

G. Hornig.

1) Valentiner, Handwörterbuch der Astronomie I, 355 (Breslau 1897).

\title{
Beobachtungen von $\mathrm{SS}=\mathrm{V} 19 \mathrm{Cygni}$. Von $A . A$. Nijland.
}

(Fortsetzung von A. N. $476 \mathrm{I}$.)

Im Jahre 1914 erhielt ich in 144 Nächten I 53 Be- nach der Stufenmethode durch Anschluß an je zwei Sterne, obachtungen des Veränderlichen $\mathrm{SS}=\mathrm{V} \cdot 19$ Cygni. Dazu kommen 8 Schätzungen des Herrn van der Bilt. Wie friiher wurde die Helligkeit im Io-Zöller $(f=319 \mathrm{~cm})$, bei den Maxima öfters auch im 3 -zölligen Sucher $(f=$ I I $3 \mathrm{~cm})$ ausnahmsweise an einen oder drei, geschätzt.

Auch jetzt weist die Beobachtungsreihe ein paar $(4)$ größere Lücken von Io bis 16 Tagen auf: es wurden keine Beobachtungen in den Intervallen Febr. I I-2 I, März $3^{-12}$, 
Aug. 20-30 und Okt. I 8 bis Nov. 2 erhalten. Trotz dieser Lücken liegt die Lichtkurve d. J. I $9{ }^{4} 4$ ziemlich fest: es ist kaum möglich, daß der Stern noch weitere Maxima als die unten mitzuteilenden $\mathrm{Nr}$. 67-73 vollzogen hätte; und nur vom Maximum $68 \mathrm{kann}$ der Typus nicht sicher angegeben werden.

Zwischen den Maxima war der Stern von nahezu kon-

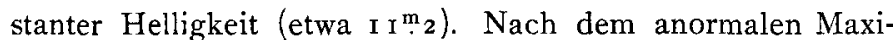
mum 72 sank er aber bis $I^{\mathrm{m}} \cdot 5$ und nach dem Maximum 73 bis $\mathrm{I} \mathrm{I}^{\mathrm{m}} \cdot 4$. Weiter möchte ich eine Aufhellung bis $\mathrm{IO}^{\mathrm{m}} \cdot 5$ (Juli $27=$ J. D. $242034 \mathrm{I}$ ) erwähnen. Es konnte leider trotz eifriger Überwachung nur diese vereinzelte Schätzung erhalten werden. Das "Monthly Report of the American Association of Variable Star Observers " gibt (Pop. Astr. 22.524) über dieses anormale Aufleuchten keinen endgültigen Aufschluß, da hier keine Beobachtungszeiten mitgeteilt werden. Aus dem augenscheinlich sehr heterogenen Material, das sehr starke Abweichungen aufweist, scheint immerhin hervorzugehen, daß die meisten amerikanischen Beobachter den Stern am 26 . Juli bedeutend $\left(0^{\mathrm{m}} 7\right.$ bis $\left.0^{\mathrm{m}} 8\right)$ heller als 8 Tage vorher sahen. Ich möchte deshalb diese Aufhellung wohl als verbürgt betrachten.

Hier folgt nun die übliche Übersicht der Maxima. Außer dem Maximum ist wieder der Moment gegeben, in dem SS Cygni beim Aufstieg die Helligkeit $9^{\mathrm{m}} \cdot 35$ erreichte. Unter "Dauer《 ist, wie früher, zu verstehen, wieviel Tage

Utrecht, I9I 5 Jan. 3 . der Veränderliche heller als I I mo war; auch die unter $» B b$. « angeführte ZahI der Beobachtungen bezieht sich auf die Helligkeit $\geq_{\mathrm{I} I}{ }^{\mathrm{m}} \mathrm{o}$. Die Zeit ist m. Z. Greenwich.

\begin{tabular}{|c|c|c|c|c|c|c|c|c|}
\hline $\mathrm{E}_{\mathrm{p}}$ & $\mathrm{SS}=9^{\mathrm{m}} \cdot 35$ & \multicolumn{3}{|c|}{ Maximum } & Gr. & $\mathrm{Bb}$. & Dauer & Bem. \\
\hline & & \multicolumn{3}{|c|}{ I9I 4} & & & & \\
\hline 67 & 2420149.0 & Jan. & $20=$ & OI 53 & $8^{m} \cdot 5$ & I I & $18^{\mathrm{d}}$ & lang \\
\hline 68 & $198 ?$ & März & $9 ?$ & $201 ?$ & $8.8 ?$ & 2 & I 2 ? & kurz? \\
\hline 69 & 253.5 & Mai & 4 & 257 & 8.5 & 10 & I9 & lang \\
\hline 70 & 317.0 & Juli & 5 & $3 \times 9$ & 8.8 & I I & I 2 & kurz \\
\hline $7 I$ & $35^{8.8}$ & Aug. & I 8 & $3^{6} 3$ & 8.7 & 10 & 19 & lang \\
\hline 72 & - & Okt. & I & 407 & $9 \cdot 3$ & I 3 & I 8 & anormal \\
\hline 73 & $440.6 ?$ & Nov. & 6 & 443 & 8.8 & 6 & I I & kurz \\
\hline
\end{tabular}

Bemerkungen. Nr. 68. Das oben zitierte "Monthly Report« gibt (Pop. Astr. 22.323) den Stern noch am 4. März schwach an. Das Maximum ist sehr wahrscheinlich kurz. Nr. 72. Der Stern vollzog in Io Tagen eine langsame Steigung von $\mathrm{I}_{\mathrm{I}}^{\mathrm{m}} \mathrm{I}$ bis $9^{\mathrm{m}} \cdot 3$, welche Tag für Tag verfolgt werden konnte. - Nr. 73. Der Aufstieg wurde nicht beobachtet. Der Stern war aber am 2. November noch schwach; das Maximum war daher wohl kurz.

Zum Schluß, sei noch bemerkt, daß ich am 25. Dezember einen ziemlich raschen Anstieg zu einem neuen Maximum beobachtete, und daß der Stern am 29. und $3 \circ$. etwa $8^{\mathrm{m}} \cdot 5$ geschätzt wurde.

\section{A. A. Nijland.}

\section{Mitteilungen über Kleine Planeten.}

Aufnahmen auf der Königstuhl-Sternwarte.

Planet Ort I9I5.0 Tägl. Bew. Gr. Platte
I 9 I 5 Febr. I 6.

29 Amphitrite $\mathrm{II}^{\mathrm{h}} 42^{\mathrm{m}} \cdot 8+4^{\circ} 2^{\prime}-\quad-\quad-\mathrm{A}_{7624}$ I 9 I 5 März 9.

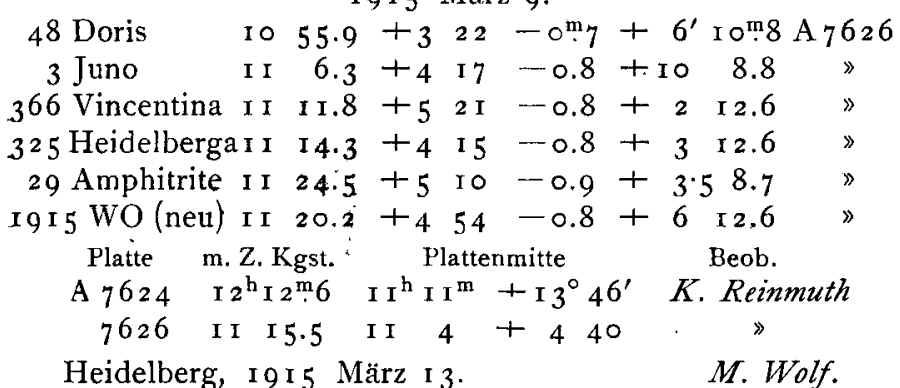

Ephemeriden-Korrektionen.

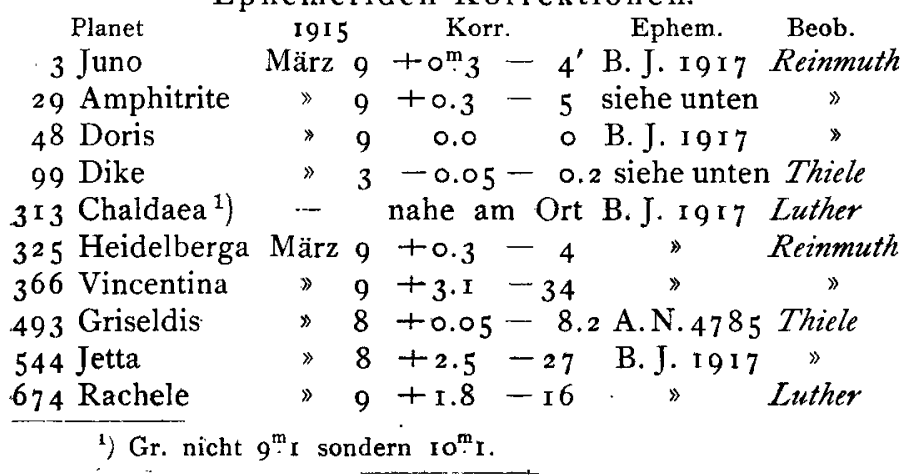

Ephemeride von 29 Amphitrite.

An Stelle der fehlerhaften Ephemeride im B. J. I 917 ist folgende zu setzen:

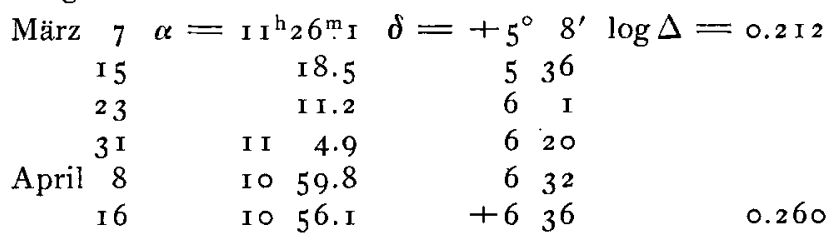

Ephemeride von 772 [rgr 3 TR]

berechnet mit neuen Elementen. $12^{\mathrm{h}} \mathrm{m}$. Z. Berlin.

\begin{tabular}{|c|c|c|c|c|c|c|c|c|}
\hline \multirow{2}{*}{\multicolumn{2}{|c|}{$\stackrel{1915}{\text { April }} 8$}} & \multicolumn{3}{|c|}{$\alpha$ vera } & \multicolumn{2}{|c|}{$\delta$ vera } & \multirow{2}{*}{$\begin{array}{c}\log r \\
0.4328\end{array}$} & \multirow{2}{*}{$\begin{array}{c}\log \Delta \\
0.2578\end{array}$} \\
\hline & & $14^{\mathrm{h}}$ & & $53^{5}$ & $+21^{\circ}$ & $57^{\prime} \cdot 1$ & & \\
\hline & I 2 & I 4 & 2 & 39 & $2 \mathrm{I}$ & 53.7 & & \\
\hline & I 6 & 13 & $5^{8}$ & 19 & $2 I$ & 45.6 & 0.4326 & 0.25 \\
\hline & 20 & I 3 & 53 & $5^{6}$ & 2 I & $3^{2.6}$ & & \\
\hline & 24 & I 3 & 49 & 35 & $2 \mathrm{I}$ & I 4.7 & 0.4325 & 0.25 \\
\hline & 28 & I 3 & 45 & 20 & 20 & 51.9 & & \\
\hline \multirow[t]{5}{*}{ Mai } & 2 & I 3 & $4 I$ & I 6 & 20 & 24.4 & 0.4324 & 0.26 \\
\hline & 6 & I 3 & 37 & 25 & r9 & $5^{2.4}$ & & \\
\hline & I0 & 13 & 33 & $5 \mathrm{I}$ & 19 & I $6 . \mathrm{I}$ & 0.4324 & 0.27 \\
\hline & I 4 & I 3 & 30 & 37 & 18 & 35.9 & & \\
\hline & 18 & 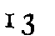 & 27 & 44 & I 7 & $5^{2.2}$ & 4 & 0.2 \\
\hline
\end{tabular}

Opp. in AR: April 20. - Gr. $1 \mathrm{x}^{\mathrm{m}} 6.6$ - Var.: $\pm \mathrm{r}^{\mathrm{m}}:$ 干 $\mathbf{3}^{\prime} \cdot \mathrm{o}$

Die neuen Elemente erfordern zur Darstellung der Beobachtungen der mit 772 identischen Planeten $1902 \mathrm{KM}$ und rq $10 \mathrm{KH}$ die Korrektionen + ro.'5 bezw. -6.5 in mittl. Anomalie. 